Do Autistic Symptoms Persist Across Time? Evidence of Substantial Change in Symptomatology Over a 3-year Period in Cognitively Able Children With Autism

\title{
Elizabeth Pellicano
}

Centre for Research in Autism and Education, London, United Kingdom; and University of Western Australia, Perth, Australia

Pellicano, E. (2012). Do autistic symptoms persist across time? Evidence of substantial change in symptomatology over a 3-year period in cognitively able children with autism. American Journal on Intellectual and Developmental Disabilities, 117, 156-166. 


\begin{abstract}
This study investigated the extent and nature of changes in symptomatology in cognitively able children with autism over a 3-year period. Thirty-seven children diagnosed with an autism spectrum condition involved in an earlier study ( $M$ age $=5$ years, 7 months $)$ were followed and reassessed 3 years later $(M$ age $=8$ years, 4 months). Scores on the Social Communication Questionnaire (SCQ; Rutter, M., Bailey, A., \& Lord, C., 2003) decreased significantly over time in all symptom domains but especially in the social domain, and correlational findings suggested the presence of 2 distinct developmental trajectories—-social communication and repetitive behaviors - that interact across time. Furthermore, 7 children (19\% of sample) made substantial changes to the extent that they failed to meet criteria on diagnostic instruments (the Autism Diagnostic Observation Schedule-Generic [ADOS-G; Lord, C., Rutter, M., DiLavore, P. C., \& Risi, S., 1999] and the SCQ) 3 years later. Children showing diagnostic discontinuity were distinguishable from those who fulfilled ADOS-G criteria only in terms of the age at which they began receiving intervention. The presence of a significant proportion of children showing considerable progress over the 3-year period challenges assumptions of diagnostic continuity and highlights the potential long-term benefits of early intervention.
\end{abstract}

Key Words: autism; longitudinal; symptoms; outcome; development 
Autism is a developmental condition defined in terms of the presence of social and communication difficulties and a range of restricted and repetitive interests and activities. In current diagnostic systems (American Psychiatric Association, 2000; World Health Organization, 1992), autism comes under the heading of "pervasive developmental disorder," implying that it is a permanent, lifelong condition-one that persists across time. These systems focus exclusively on behaviors in the young child; the early emergence of autistic features (before 3 years of age) is one of the key criteria for an autism diagnosis, and rediagnosis at a later point during development is rare in clinical practice.

This view, however, presents a rather static picture of autism. Indeed, the assumption of continuity in symptomatology is not supported fully by the few longitudinal studies that have traced the development of autism. Although long-term outcome studies have confirmed that the behavioral features of autism generally endure through childhood into adulthood (e.g., Howlin, Goode, Hutton, \& Rutter, 2004; Howlin, Mawhood, \& Rutter, 2000; Kanner, 1973; Rutter, Greenfeld, \& Lockyer, 1967; Szatmari, Bartolucci, Bremner, Bond, \& Rich, 1989), they also have reported considerable changes over time in the manifestation of autistic symptoms (e.g., Howlin et al., 2000; Sigman \& Ruskin, 1999). Furthermore, these studies consistently have highlighted the striking variability in long-term outcomes of autistic individuals. The majority of individuals are unable to live independently or enjoy friendships and social interactions, but there is a significant proportion of individuals (10\%-28\%; Howlin et al., 2000, 2004; Szatmari et al., 1989; Venter, Lord, \& Schopler, 1992; although see Farley et al., 2009) who attain "good" outcomes, achieving independence and gaining qualifications. The presence of at least some 
individuals who go on to lead relatively well-adjusted lives is prima facie evidence of discontinuity in autistic symptomatology.

These longitudinal findings raise two important questions: Does a child diagnosed with autism necessarily remain with the condition throughout his or her life course? And what are the characteristics that distinguish children who make marked behavioral improvements over time from those who do not? The few early attempts to answer these questions showed that most, but not all, preschoolers diagnosed with an autism spectrum disorder (ASD) retain a spectrum diagnosis in late childhood, adolescence, and early adulthood. For example, in Rutter et al.'s (1967) study, 54 out of $63(86 \%)$ children diagnosed with infantile psychosis (i.e., autism) at 5 years showed sufficient symptoms to be termed autistic at follow-up 5-15 years later. Similarly, Sigman and Ruskin (1999) reported that 45 out of 50 children with autism (90\%) met current criteria for diagnosis on the Autism Diagnostic Interview (ADI; Le Couteur et al., 1989) when reassessed in middle childhood (age 12 years), and 40 out of 44 of these same individuals (91\%) met ASD criteria on the Autism Diagnostic Observation Schedule - Generic (ADOS-G; Lord, Rutter, DiLavore, \& Risi, 1999) when reexamined in late adolescence (McGovern \& Sigman, 2005). Investigations of the factors that influence long-term outcomes have identified an IQ above 50 and the presence of meaningful speech at 5-6 years as important prognostic indicators (Lord \& Bailey, 2002).

Recently, attention has been directed toward the diagnostic continuity of children identified very early on. Overall, the consensus is that a diagnosis of an ASD at age 2 years withstands the test of time when reevaluated over both short (1-2 years: Lord, 1995; Stone et al., 1999) and longer (5 years: Charman et al., 2005; 7 years: Lord et al., 2006; Turner, Stone, Pozdol, \& Coonrod, 2006) durations. However, 
findings have also demonstrated that, in agreement with those from long-term outcome studies, a significant minority of children (up to 37\%) show abatement of symptoms to the extent that they no longer meet criteria for a spectrum diagnosis (Charman et al., 2005; Eaves \& Ho, 2004; Lord et al., 2006; Turner et al., 2006; Turner \& Stone, 2007). These studies have identified, furthermore, a range of largely intrinsic factors that might predict diagnostic discontinuity. Children who failed to meet diagnostic criteria at follow-up were likely to have better verbal and nonverbal IQ at age 2 years (Lord et al., 2006; Turner et al., 2006) or age 7 years (Charman et al., 2005) and engage in fewer repetitive behaviors at age 2 (Lord et al., 2006). In addition, Turner et al. (2006) reported that children who received more hours of speech and language therapy between 2 and 3 years of age were less likely to reach diagnostic criteria for autism at follow-up than those who did not. These latter findings are particularly noteworthy because they show evidence of the positive effect of early intervention on children's developmental outcomes, an issue little investigated in existing studies.

The findings from short-term outcome studies, therefore, have generally supported those from longer term studies. A significant proportion of autistic children do not show sufficient symptoms to warrant a diagnosis in late childhood, and these children tend to have better verbal and nonverbal ability and milder levels of symptomatology at diagnosis than children who continue to meet diagnostic criteria. These follow-up studies, however, included individuals who varied widely in IQ and degree of symptomatology, rendering it unclear whether these same factors influence the developmental outcomes of cognitively able, or so-called high-functioning autistic children for whom there is considerable potential for independence. The few longterm, follow-up studies that have focused specifically on individuals of at least 
average intellectual functioning have revealed that adult outcomes are highly variable even within this group (Howlin et al., 2004; Szatmari et al., 1989; Venter et al., 1992), but the evidence is mixed concerning precisely which factors explain this variability. As such, the factors that contribute to marked changes in symptomatology in more able autistic individuals — both in the shorter and longer terms — remain largely unknown.

Even if children continue to meet criteria for autism, the nature of their symptoms - in social reciprocity, communication, and repetitive behaviors - is susceptible to change over time. A few prospective studies have examined this issue. Starr, Szatmari, Bryson, and Zwaigenbaum (2003) administered the Autism Diagnostic Interview—Revised (ADI-R; Lord, Rutter, \& Le Couteur, 1994) to parents of cognitively able children with autism $(n=41)$ and Asperger syndrome $(n=17)$ at intake (aged 4-6 years) and again 2 years later. They found little change over time in repetitive behaviors for either group. In contrast, communication symptoms improved significantly for children with autism but not for children with Asperger syndrome, whereas social symptoms worsened over time for both groups, but particularly for children with Asperger syndrome.

Charman et al. (2005) administered the ADI-R to parents of 26 children with autism at ages $2,3,4-5$, and 7 years. They found significant improvements in symptomatology over time, but the pattern of change varied according to symptom domain. Social symptoms abated between age 4-5 years and age 7 , and nonverbal communication improved across all time points. Repetitive behaviors deteriorated between 3 years and age 4-5 years but improved from age $4-5$ years to age 7 years. These findings, like those from retrospective studies (Fecteau, Mottron, Berthiaume, \& Burack, 2003; Piven, Harper, Palmer, \& Arndt, 1996), suggested that each 
symptom domain follows a distinct developmental course. This notion agrees with Happé, Ronald, and Plomin's (2006) proposal that the core features of autism might be separable and genetically distinct. In partial support of this idea, Charman et al. (2005) reported that scores on the repetitive behaviors domain at age 3 were not significantly related to scores on the social and communication domains at age 7 . Yet, these authors also found that age 3 social and communication scores were significantly associated with such scores at age 7 , suggesting that these aspects of autism might in fact be related throughout development (see, also, Lord et al., 2006).

Overall, the findings of existing longitudinal studies have indicated a pattern of change, rather than of continuity, despite the standard diagnostic assumption to the contrary. The majority of autistic children display changes in the expression of behavioral symptoms, yet the nature of those changes is far from certain, and it is unclear whether the changes reflect different developmental pathways for each symptom domain or whether they might be interrelated over time. Moreover, a proportion of children make considerable progress, but the reasons for why marked improvements take place in some children and not in others remain unclear, at least in cognitively able children with autism. Such knowledge is critical not only for achieving a complete etiological understanding of the condition but also for delineating ways to help maximize children's developmental outcomes.

The current longitudinal study tested the assumption of continuity in symptomatology in cognitively able children over a 3-year period. The first aim was to determine the extent of changes in symptomatology and to pinpoint which factors, including intrinsic (early verbal and nonverbal ability, initial symptomatology) and extrinsic (intervention) characteristics, potentially contribute to variability in children's behavioral outcomes. The second aim was to specify the nature of changes 
in each core symptom domain and to determine whether this pattern differed according to behavioral domain. The final aim was to elucidate any developmental links between symptom domains.

\section{Method}

\section{Participants}

Forty-five children with an ASD (40 boys) who were from 4 to 7 years old ( $M$ age $=$ 67.5 months, $S D=10.3$ ) participated in the initial study (Time 1; Pellicano, Maybery, Durkin, \& Maley, 2006). All children had received an independent clinical diagnosis of either autism ( $n=30 ; 67 \%$ of sample), pervasive developmental disorder-not otherwise specified (PDD-NOS; $n=12 ; 27 \%)$, or Asperger syndrome $(n=3 ; 6 \%)$, according to Diagnostic and Statistical Manual of Mental Disorders (4th edition) criteria (DSM-IV; American Psychiatric Association, 1994), and the Autistic Diagnostic Interview—Revised (ADI-R; Lord et al., 1994) by a multidisciplinary team. At intake, no child had a coexisting medical or developmental condition as reported by parents or obtained a verbal or nonverbal IQ score less than 80. Most of the children were White, and the parents were of mixed socioeconomic backgrounds. All children spoke English as their first language.

Thirty-seven out of 45 children with autism ( $84 \%$ of original sample) were available for reassessment 3 years later (Time 2; $M$ age $=100.13$ months; $S D=11.4$; range $=80-119$ ), including 26 children with autism, 9 with PDD-NOS, and 2 with Asperger syndrome. The mean time between the initial and follow-up studies was 32.9 months. Of the 8 families who were unavailable for reassessment, 2 had relocated to another state, and 6 families were untraceable. The 37 children with autism (33 boys) represented the original sample well: No significant differences were found among those children who were not reassessed $(n=8)$ and those in the follow- 
up group $(n=37)$ in terms of age $(p=.60)$, verbal ability $(p=.43)$, nonverbal ability $(p=.27)$, or ADI-R algorithm score $(p=.18)$.

\section{Measures}

Autistic symptomatology. The ADI-R (Lord et al., 1994) was administered to parents at Time 1 by an experienced clinician blind to the experimental aims of this study and to the children's clinical diagnoses. The ADI-R is a standardized, semistructured interview with the child's caregiver designed to obtain information about past and current behaviors characteristic of pervasive developmental disorders (PDD). For the present purposes, the algorithm and cut-off scores specified by Lord et al. (1994) were applied to ratings of current behavior (see also Charman et al., 2005). Children meeting or exceeding cut-off scores in all three domains were classified as having autistic disorder, whereas children who partially met the criterion (falling within 1-2 points on social and/or communication domains; Risi et al., 2006) were classified as being on the autism spectrum.

The Autism Diagnostic Observation Schedule-Generic (ADOS-G; Lord et al., 1999) was administered to all children at follow-up. The ADOS-G is a standardized observational scale designed to assess current autistic symptomatology. It is administered by a trained examiner and does not rely on parent report. Module 3 was administered to 33 children who were verbally fluent. Module 2 was administered to 4 children whose expressive language consisted of phrase speech. The published algorithms and cut-off scores for Autism, Autism Spectrum, and Nonspectrum were applied to the ADOS-G data; higher algorithm scores indicate greater levels of autistic symptomatology.

To assess interrater reliability, one other experienced and independent rater coded a random selection of the videotaped observations $(n=13)$. Intraclass 
correlation coefficients were computed for algorithm scores for each domain, indicating excellent agreement: Communication $=.93$, and Socialization $=.98$. Agreement for ASD/Nonspectrum distinctions was $100 \%$.

The ADI-R and ADOS-G are different diagnostic measures administered at different time points. To ensure continuity, the Social Communication Questionnaire (SCQ; Rutter et al., 2003), was administered to parents at both time points to (a) examine potential changes in children's symptomatology over time and (b) provide an index of parent-rated symptomatology at Time 2 to complement information provided by the ADOS-G. The SCQ's 40 items are derived from the well-validated ADI (Le Couteur et al., 1989), with which it demonstrates good agreement (Corsello et al., 2007). The current version was used in this study, which focuses on the presence of autistic behaviors within the previous 3 months. Scores are summed to yield a total score out of 39; higher scores indicate an elevated level of autistic symptoms, with a score $\geq 15$ as the cut-off point for a possible autism spectrum condition. The SCQ also yields scores for each symptom domain: social interaction (maximum score $=15$ ), communication (maximum score $=13$ ), and repetitive behavior $($ maximum score $=8$; see Rutter et al., 2003).

Other background assessments. At both time points, the Peabody Picture Vocabulary Test—Third Edition (PPVT-III; Dunn \& Dunn, 1997) assessed receptive language ability and the Leiter International Performance Scale-Revised (Leiter-R; Roid \& Miller, 1997) indexed nonverbal ability (see Pellicano et al., 2006, for details). At follow-up, parents were asked to complete retrospectively a brief questionnaire relating to intervention, specifying the frequency, duration, and type of intervention during both the past 3 years and earlier. Parents' responses were 
summarized as mean number of hours per week of treatment since study intake and the age at which children first began intervention.

\section{Procedures}

Ethical approval was granted by the University of Western Australia's Human Research Ethics Committee, and parents of all the children gave informed written consent prior to participation. At each time point, children were assessed on two separate occasions, each lasting $1-1.5 \mathrm{hr}$, either at the family home or at the university.

\section{Results}

\section{Background Data}

Descriptive information is provided in Table 1. All children were attending mainstream schools at follow-up, reflecting the prevailing policy of inclusion by Australian educational authorities (see Forbes, 2007), with the exception of three children, one of whom attended a special unit for children with learning disabilities and two of whom were home schooled. All children in mainstream provision were receiving extra educational support of varying degrees. In addition, according to the parents' reports, all children had received intervention services previously, which began at a mean age of 39.2 months $(S D=13.4$; range $=16-66$ months $)$. Thirty-two children had received intervention (albeit nonintensive) during the past 3 years at a median rate of $1.4 \mathrm{hr}$ of intervention per week (interquartile range $=3.1$ ) of either applied behavioral analysis $(n=5)$, speech and language therapy $(n=5)$, group-based social skills training $(n=3)$, or a combination of these approaches $(n=19)$.

All of the 37 children had a preexisting clinical diagnosis of an autism spectrum condition. The ADI-R administered at Time 1 confirmed those diagnoses. 
Of the 37 children assessed at Time 1, 25 children met full ADI-R diagnostic criteria for autism, and 12 children met criteria for the broader classification of ASD.

\section{Diagnostic Outcomes}

Children's diagnostic outcomes were ascertained by examining children's ADOS-G classifications at Time 2 (see Turner et al., 2006, for similar use of the ADOS-G as the primary outcome measure). Among the 25 children who fulfilled ADI-R criteria for autism at Time 1,18 children met ADOS-G criteria for autism at follow-up, and 2 children met criteria for "autism spectrum," whereas of the 12 children who met partial ADI-R criteria at Time 1,10 met criteria for autism $(n=9)$ or autism spectrum $(n=1)$ on the ADOS-G at Time 2 . Some of the discordant crosstime diagnoses - particularly the crossing of diagnostic categories (e.g., from autism to not autistic) — might be expected from the use of different instruments at intake (ADI-R) and follow-up (ADOS-G). For this reason, additional information from the SCQ was examined in detail below (see Corsello et al., 2007). However, what is of key interest here is the number of children who showed diagnostic discontinuity - that is, those who failed to reach the autism spectrum threshold at Time 2 despite having an existing clinical and ADI-R diagnosis of an autism spectrum condition (see Figure $1)$.

Overall, 30 out of the 37 children retained an autism spectrum diagnosis according to ADOS-G criteria at Time 2. Seven children (19\% of sample; age range: 7 years, 0 months-9 years, 11 months; 5 children with clinical diagnoses of autism, 2 with PDD-NOS), however, failed to meet ADOS-G criteria for an autism spectrum diagnosis at follow-up. Generally, these children were verbally fluent, could converse in a back-and-forth manner, and used eye gaze and gestures to modulate the interaction. There was, nonetheless, an odd quality about the interaction. Children's 
attempts to initiate social interaction were limited; their social responsiveness was somewhat awkward; and social reciprocity was restricted in frequency. Indeed, all 7 children met the cut off for ASD in one of the two ADOS-G domains (social only: $n=$ 6; communication only: $n=1$ ), reflecting clinically significant difficulties (Lord et al., 1999).

Parental reports of symptomatology at follow-up (indexed by SCQ scores) corroborated the diagnostic outcomes of these nonspectrum children: All children obtained current SCQ scores below the cut-off score of 15 for ASD (range of scores = 2-11). Comparisons between outcome groups (ASD vs. nonspectrum) on total SCQ scores showed that nonspectrum children obtained significantly lower SCQ scores at follow-up than children who showed diagnostic continuity, $t(34)=4.38, p<.001$, but that such differences were not present at intake, $t(34)<1$ (see Table 1 for means).

These two groups were compared further on those variables identified previously as important determinants of outcome (see Table 1). Because of the number of comparisons made, results were not considered significant unless they reached $p$ values of less than .01. Contrary to previous reports, nonspectrum children were no different from their 30 peers who fulfilled ADOS-G criteria in terms of receptive language ability at intake or follow-up, initial symptom levels (ADI-R scores), or number of hours per week of intervention received in the 3-year period (all $p s>.47)$. Comparisons on early nonverbal ability approached significance, $F(1,35)=$ $3.10, p=.08$, but the group means were in the opposite direction expected. Nonspectrum children showed, on average, lower nonverbal ability scores at intake than children who met ADOS-G criteria. There was one characteristic, however, that successfully distinguished the groups: the age at which children began receiving intervention. On average, nonspectrum children received services significantly earlier 
$(M=27.9$ months, $S D=7.9)$ than those who retained a spectrum diagnosis at followup: $M=41.9, S D=13.1, F(1,35)=7.47, p=.009$.

\section{Continuity in Autistic Symptomatology}

To examine changes over time in symptomatology, SCQ domain scores were calculated for both time points for the entire group. A repeated measures analysis of variance (ANOVA) on SCQ scores, with time and domain (social, communication, repetitive behaviors) as factors, revealed significant main effects of time, $F(1,35)=$ 49.14, $p<.001$, and domain, $F(2,70)=9.67, p<.001$, and a significant Time $\times$ Domain interaction, $F(2,70)=14.43, p<.001$. Children's symptomatology improved considerably over time, but the magnitude of change over time varied by symptom domain. Improvement was greater in the area of social interaction (Time 1: $M=8.95$, $S D=4.10$; Time $2: M=4.58, S D=3.16$ ) than in the areas of communication (Time 1: $M=7.27, S D=2.10 ;$ Time $2: M=5.39, S D=1.96), t(35)=4.27, p<.001$, and repetitive behaviors (Time 1: $M=5.89, S D=2.04$; Time $2: M=4.17, S D=2.10$ ), $t(35)=4.68, p<.001$. There was no difference in the amount of change over time between the latter two domains, $t(35)<1$.

To examine whether the developmental trajectories of symptom domains are independent (Happé et al., 2006) or related, cross-time correlational analyses were conducted on children's SCQ domain scores (see Table 2). Again, correlation coefficients were not considered significant unless they attained $p$ values $<.01$. As expected, SCQ scores at Time 1 were significantly (positively) associated with Time 2 SCQ scores within each symptom domain: social, $r(35)=.73, p<.001$; communication, $r(35)=.38, p=.01$; and repetitive behaviors, $r(35)=.54, p<.001$. Table 2 also shows the longitudinal correlations across domains. Raw correlational analyses revealed a significant positive relationship between Time 1 social domain 
scores and Time 2 scores on the communication, $r(35)=.58, p<.001$, and repetitive behaviors, $r(35)=.38, p=.01$, domains. When variance attributable to Time 1 verbal and nonverbal ability was partialled out, social domain scores at Time 1 remained significantly correlated with Time 2 communication, $r(31)=.53, p<.001$, and repetitive behaviours scores, $r(31)=.38, p=.02$. Early repetitive behaviors, however, were unrelated to social and communication symptoms at follow-up.

\section{Discussion}

\section{Diagnostic (Dis)continuity}

The results showed that the majority of children with autism ( $81 \%$ of sample) displayed continuity over a 3-year period. Of the children who had both received independent clinical diagnoses of autism or related conditions and reached diagnostic algorithm thresholds on the ADI-R, there was a significant minority of children (one fifth of the sample) who experienced substantial improvements in symptomatology: Seven children failed to reach criteria for an autism spectrum diagnosis at follow-up. This figure is consistent with those of previous studies (e.g., Charman et al., 2005; Rutter et al., 1967; Sigman \& Ruskin, 1999).

One straightforward explanation for children's unstable diagnoses might be that they were misclassified as nonspectrum by the ADOS-G. Although the ADOS-G is effective at differentiating children who definitely have autism from those who do not, it shows reduced sensitivity and specificity for classifications of children with milder symptoms (Bishop \& Norbury, 2002; Lord et al., 1999; Risi et al., 2006). Nevertheless, we are confident in the current outcome data because parents' reports of their child's symptoms mapped onto the results from the ADOS-G diagnoses at Time 2. Those children who did not meet ADOS-G criteria at Time 2 also failed to show 
sufficient autistic symptoms on the SCQ at that time, although that same group had met criteria for ASD on both the ADI-R and the SCQ at Time 1.

These children, then, might have experienced genuine diagnostic discontinuity over the 3-year period. Previous reports have implicated various child characteristics that contribute to unstable diagnoses (e.g., Charman et al., 2005; Lord et al., 2006; Turner et al., 2006). In the present study, however, child factors, including verbal and nonverbal ability at intake or follow-up and early symptomatology, were unable to distinguish children who showed diagnostic instability from those who did not. Differences in sample selection might underlie the discrepancy between previous studies, which included children who varied widely in ability and degree of symptomatology (Charman et al., 2005; Lord et al., 2006; Sigman \& Ruskin, 1999; Turner et al., 2006), and the present study, which included only children of (at least) average ability. Indeed, the current findings are consistent with those of Szatmari, Bryson, Boyle, Streiner, and Duku (2003), who found that initial verbal and nonverbal IQ scores were a reasonably poor predictor of later social and communicative symptoms in cognitively able children with autism and Asperger syndrome. Together, these findings raise the possibility that the results from outcome studies including less able children with autism might not be generalizable to cognitively able children with an autism spectrum condition.

Notably, one factor distinguished children showing different diagnostic outcomes. The 7 children showing the most progress were those children who began receiving behavioral intervention from a very young age ( $M=28$ months $)$ - on average, 14 months earlier than those children who made relatively less improvement over time. Harris and Handleman (2000) reported similar findings: Children who began an intensive behavioral intervention program before 42 months of age were 
more likely to be placed in a mainstream setting than a special education setting compared with children who began the program at, on average, 54 months of age.

Together these findings attest to the effectiveness of early behavioral intervention. They are, nonetheless, potentially counterintuitive: One might have expected those who received intervention very early on to be those children who had especially severe and obvious symptoms. The fact that the SCQ scores of the two outcome groups were equivalent at intake, but not at follow-up, suggests further that the effects of early intervention might not be immediate but might lie dormant for a certain period. These findings are certainly intriguing. Caution is warranted, given the limited information collected regarding intervention and the retrospective nature of the data, which make it impossible to determine the specific effects of specific treatments. The findings nevertheless highlight the importance of systematically collecting data on early intervention services in prospective studies of autism.

If one accepts that these children genuinely showed insufficient autistic features to meet current instrument criteria, should this mean that they "recovered" (see Helt et al., 2008)? There are three reasons to be wary here. First, these children displayed core autism symptoms prior to age 36 months. According to current diagnostic systems, which underscore lifetime criteria, these children would be considered to have autism (although nosological discussion may be warranted; Charman, 2003). Second, it is not that these children did not show any autistic features. They showed clinically significant difficulties in one ADOS-G domain, predominantly in the social domain. These findings suggest that there was some continuity in specific aspects of autism that reinforce the lifelong nature of the condition. 
Third, the ADOS-G does not provide absolute levels of symptomatology: Target behaviors are coded relative to the child's developmental level. Therefore, although these 7 children fell short of the cut-off criteria at this particular point during development, they might not do so during subsequent assessments. As social interactions become more demanding as children develop, it is possible that their symptoms might appear more "autistic" over time relative to mental-age-matched peers. Charman et al. (2005) found that diagnostic classifications changed considerably over a 5-year period, with children crossing (and re-crossing) diagnostic boundaries. These findings highlight the need both to trace changes in symptomatology at multiple points across time and to continue intervention even when children show improvement.

\section{Continuity in Autistic Symptomatology}

In this study, we found clear evidence of change over time in symptomatology, which is entirely consistent with previous reports. What is less consistent, however, is that significant progress was made in all three symptom domains and that progress was greatest in the area of social reciprocity. Charman et al. (2005) found evidence of progress in children's social interaction and communication but no concomitant improvement in behavioral flexibility. Starr et al. (2003) also reported no significant lessening of repetitive behaviors over time but that social symptoms increased over time. It is unlikely that this discrepancy in findings resulted from differences in indices of symptomatology (SCQ vs. ADI-R) because the SCQ covers behaviors similar to those covered in the ADI-R.

The substantial improvement in social scores relative to the other two symptom domains could result from the large range of possible scores in the social domain, providing the greatest opportunity to observe improvements over time. One 
alternative explanation for the considerable progress across domains, but especially in the social domain, might lie with the fact that almost all children were placed in regular, mainstream classrooms. Cognitively able children show more peer engagement than less able autistic children (Sigman \& Ruskin, 1999), and it is possible that typical children might have served as role models for appropriate social interaction. Consistent with this possibility, Lord and Hopkins (1986) demonstrated that, following 6 weeks of regular unstructured play sessions with typical children, school-aged children with autism showed an increase in the amount of time watching their typical peers and increased responsiveness to play invitations, combined with a decrease in the amount of self-stimulatory behaviors. Thus, children in the current study might have benefited from regular opportunities to interact with typical children.

\section{Longitudinal Associations Among Symptom Domains}

Previous studies have suggested that the different symptom domains might take different developmental courses (Charman et al., 2005; Lord et al., 2006; Piven et al., 1996). In the present study, individual differences in social and communication symptoms were interlinked both concurrently and longitudinally, whereas early repetitive behaviors were not predictive of social and communication skills at followup. These findings support the possibility that the social and communication domains might be a unitary symptom domain (consistent with proposed Diagnostic and Statistical Manual of Mental Disorders [forthcoming, 5th edition] guidelines for autism; American Psychiatric Association, 2010) that follows a distinct developmental trajectory from the repetitive behaviors domain (Lord et al., 2006). Furthermore, fewer social symptoms during early development predicted not only fewer communicative symptoms 3 years later but also less repetitive behavior. This 
latter finding indicates that the degree of early social difficulties potentially influences the development of symptoms in other domains. Whether all three developmental trajectories are distinct from the outset (see Happé et al., 2006) and interact as development unfolds and whether the social and communication symptom domains are in fact overlapping are important questions for future research.

\section{Conclusion}

This study showed discontinuity in symptomatology over a 3-year period in a moderate-sized sample of cognitively able children with autism, challenging the prevailing static picture of autism. It is encouraging that the figure for diagnostic discontinuity here (19\%) is similar to figures from previous studies and that the ADOS-G nonspectrum diagnoses converged with information obtained via parent report. The results offer reasons for optimism: A significant proportion of children improved to the extent that they did not meet instrument criteria for a spectrum diagnosis at follow-up, and early intervention was the only factor that distinguished these children from those who continued to meet criteria. Having demonstrated these positive findings in a community-based sample, it will be important that they be replicated in a clinic-based sample. Tracking these children as they approach adolescence will be crucial for establishing whether the extent and nature of improvements in symptomatology continue over time and whether the developmental trajectories of symptom domains remain interconnected or take diverging developmental courses. 


\section{References}

American Psychiatric Association. (1994). Diagnostic and statistical manual of mental disorders (4th ed.). Washington, DC: Author.

American Psychiatric Association. (2000). Diagnostic and statistical manual of mental disorders (4th ed., text rev.). Washington, DC: Author.

American Psychiatric Association. (2010). A 09 autism spectrum disorder. Retrieved from

http://www.dsm5.org/ProposedRevisions/Pages/proposedrevision.aspx?rid=94

Bishop, D. V. M., \& Norbury, C. F. (2002). Exploring the borderlands of autistic disorder and specific language impairment: A study using standardised diagnostic instruments. Journal of Child Psychology and Psychiatry, 43, $917-$ 929.

Charman, T. (2003). Epidemiology and early identification of autism: Research challenges and opportunities. In Autism: Neural basis and treatment possibilities, Novartis Foundation Symposium 251 (pp. 10-19). Chichester, UK: Wiley.

Charman, T., Taylor, E., Drew, A., Cockerill, H., Brown, J. A., \& Baird, G. (2005). Outcome at 7 years of children diagnosed with autism at age 2: Predictive validity of assessments conducted at 2 and 3 years of age and pattern of symptom change over time. Journal of Child Psychology and Psychiatry, 46, $500-513$.

Corsello, C., Hus, V., Pickles, A., Risi, S., Cook, E. H., Leventhal, B. L., \& Lord, C. (2007). Between a ROC and a hard place: Decision making and making decisions about using the SCQ. Journal of Child Psychology and Psychiatry, 48, 932-940. 
Dunn, L. M., \& Dunn, D. M. (1997). Peabody Picture Vocabulary Test-Third edition. Circle Pines, MN: American Guidance Service.

Eaves, L. C., \& Ho, H. H. (2004). The very early identification of autism: Outcome to age 4 1/2-5. Journal of Autism and Developmental Disorders, 34, 367-378.

Farley, M. A., McMahon, W. M., Fombonne, E., Jenson, W. R., Miller, J., Gardner, M., ... Coon, H. (2009). Twenty-year outcome for individuals with autism and average or near-average cognitive abilities. Autism Research, 2, 109-118.

Fecteau, S., Mottron, L., Berthiaume, C., \& Burack, J. (2003). Developmental changes of autistic symptoms. Autism, 7, 255-268.

Forbes, F. (2007). Towards inclusion: An Australian perspective. Support for Learning, 22, 66-71.

Happé, F., Ronald, A., \& Plomin, R. (2006). Time to give up on a single explanation for autism. Nature Neuroscience, 9, 1218-1220.

Harris, S. L., \& Handleman, J. S. (2000). Age and IQ at intake as predictors of placement for young children with autism: A four- to six-year follow-up. Journal of Autism and Developmental Disorders, 30, 137-142.

Helt, M., Kelley, E., Kinsbourne, M., Pandey, J., Boorstein, H., Herbert, M., \& Fein, D. (2008). Can children with autism recover? If so, how? Neuropsychology Review, 18, 339-366.

Howlin, P., Goode, S., Hutton, J., \& Rutter, M. (2004). Adult outcome for children with autism. Journal of Child Psychology and Psychiatry, 45, 212-229.

Howlin, P., Mawhood, L., \& Rutter, M. (2000). Autism and developmental receptive language disorder-A follow-up comparison in early adult life. II: Social, behavioural and psychiatric outcomes. Journal of Child Psychology and Psychiatry, 41, 561-578. 
Kanner, L. (1973). Childhood psychosis: Initial studies and new insights. New York: Winston/Wiley.

Le Couteur, A., Rutter, M., Lord, C., Rios, P., Robertson, S., Holdgrafer, M., \& McLennan, J. (1989). Autism Diagnostic Interview: A standardized investigator-based instrument. Journal of Autism and Developmental Disorders, 19, 363-387.

Lord, C. (1995). Follow-up of two-year-olds referred for possible autism. Journal of Child Psychology and Psychiatry, 36, 1365-1382.

Lord, C., \& Bailey, A. (2002). Autism spectrum disorders. In M. Rutter \& E. Taylor (Eds.), Child and adolescent psychiatry: Modern approaches (4th ed., pp. 636663). Oxford: Blackwell Scientific.

Lord, C., \& Hopkins, J. M. (1986). The social behaviour of autistic children with younger and same-age non-handicapped peers. Journal of Autism and Developmental Disorders, 16, 249-262.

Lord, C., Risi, S., DiLavore, P., Shulman, C., Thurm, A., \& Pickles, A. (2006). Autism from two to nine. Archives of General Psychiatry, 63, 694-701. Lord, C., Rutter, M., DiLavore, P. C., \& Risi, S. (1999). Autism Diagnostic Observation Schedule-Generic. Los Angeles: Western Psychological Services.

Lord, C., Rutter, M., \& Le Couteur, A. (1994). Autism Diagnostic InterviewRevised. Journal of Autism and Developmental Disorders, 24, 659-685.

McGovern, C. W., \& Sigman, M. (2005). Continuity and change from early childhood to adolescence in autism. Journal of Child Psychology and Psychiatry, 46, 401-408. 
Pellicano, E., Maybery, M., Durkin, K., \& Maley, A. (2006). Multiple cognitive capabilities/deficits in children with an autism spectrum disorder: "Weak" central coherence and its relationship to theory of mind and executive control. Development and Psychopathology, 18, 77-98.

Piven, J., Harper, J., Palmer, P., \& Arndt, S. (1996). Course of behavioural change in autism: A retrospective study of high-IQ adolescents and adults. Journal of the American Academy of Child and Adolescent Psychiatry, 35, 523-529.

Risi, S., Lord, C., Gotham, K., Corsello, C., Chrysler, C., Szatmari, P., ... Pickles, A. (2006). Combining information from multiple sources in the diagnosis of autism spectrum disorders. Journal of the American Academy of Child and Adolescent Psychiatry, 45, 1094-1102.

Roid, G. H., \& Miller, L. J. (1997). Leiter International Performance Scale-Revised. Chicago: Stoelting.

Rutter, M., Bailey, A., \& Lord, C. (2003). Social Communication Questionnaire. Los Angeles: Western Psychological Services.

Rutter, M., Greenfeld, D., \& Lockyer, L. (1967). A five to fifteen year follow-up study of infantile psychosis. II. Social and behavioural outcome. British Journal of Psychiatry, 113, 1183-1199.

Sigman, M., \& Ruskin, E. (1999). Continuity and change in the social competence of children with autism, Down syndrome, and developmental delays. Monographs of the Society for Research in Child Development, 64, 1-114.

Starr, E., Szatmari, P., Bryson, S., \& Zwaigenbaum, L. (2003). Stability and change among high-functioning children with pervasive developmental disorders: A 2-year outcome study. Journal of Autism and Developmental Disorders, 33, $15-22$. 
Stone, W. L., Lee, E. B., Ashford, L., Brissie, J., Hepburn, S. L., Coonrod, E. E., ... Weiss, B. H. (1999). Can autism be diagnosed accurately in children under 3 years? Journal of Child Psychology and Psychiatry, 40, 219-226.

Szatmari, P., Bartolucci, G., Bremner, R. S., Bond, S., \& Rich, S. (1989). A follow-up study of high functioning autistic children. Journal of Autism and Developmental Disorders, 19, 213-226.

Szatmari, P., Bryson, S. E., Boyle, M. H., Streiner, D. L., \& Duku, E. (2003). Predictors of outcome among high-functioning children with autism and Asperger syndrome. Journal of Child Psychology and Psychiatry, 44, 520528.

Turner, L. M., \& Stone, W. L. (2007). Variability in outcome for children with an ASD diagnosis at age 2. Journal of Child Psychology and Psychiatry, 48, 793-802.

Turner, L. M., Stone, W. L., Pozdol, S. L., \& Coonrod, E. E. (2006). Follow-up of children with autism spectrum disorders from age 2 to age 9. Autism, 10, 257279.

Venter, A., Lord, C., \& Schopler, E. (1992). A follow-up study of high-functioning autistic children. Journal of Child Psychology and Psychiatry, 33, 489-507.

World Health Organization. (1992). The ICD-10 classification of mental and behavioral disorders: Clinical descriptions and diagnostic guidelines. Geneva, Switzerland: Author. 
Received 10/11/2011, accepted 1/31/2012.

Editor-in-Charge: Gaia Scerif

Funds from the British Academy, the Experimental Psychology Society, and University College, Oxford, supported this research. The author is grateful to all participating families, to Alana Maley-Berg, Courtenay Norbury, and Simon Wallace for assistance with coding, and to Dorothy Bishop, Murray Maybery, and Marc Stears for helpful discussions and comments on a previous draft. Research at the Centre for Research in Autism and Education is supported by The Clothworkers' Foundation and Pears Foundation.

Correspondence regarding this article should be sent to Elizabeth Pellicano, Centre for Research in Autism and Education, Department of Psychology and Human Development, Institute of Education, University of London, 25 Woburn Square,

London WC1H 0AA, United Kingdom. E-mail: l.pellicano@ioe.ac.uk 
Table 1

Descriptive Characteristics at Time 1 and Time 2 for the Autism Spectrum Disorder (ASD) Group and for Outcome Subgroups of Children Who Either Fulfilled ( $\mathrm{n}=30)$ or Did Not Fulfill $(\mathrm{n}=7)$ Autism Diagnostic Observation Schedule-Generic Criteria for an ASD Diagnosis at Follow-up

\begin{tabular}{|c|c|c|c|c|}
\hline \multirow[b]{2}{*}{ Time } & \multirow[b]{2}{*}{ Variable } & \multirow[b]{2}{*}{$\begin{array}{l}\text { Total } \\
(n=37): \\
M(S D) \\
\text { Range }\end{array}$} & \multicolumn{2}{|c|}{ Outcome subgroups } \\
\hline & & & $\begin{array}{c}\text { ASD } \\
(n=30): \\
M(S D) \\
\text { Range }\end{array}$ & $\begin{array}{c}\text { Nonspectrum }(n \\
=7): \\
M(S D) \\
\text { Range }\end{array}$ \\
\hline \multirow[t]{10}{*}{ Time 1} & Age (months) & $67.92(10.42)$ & $66.67(9.39)$ & $73.28(13.56)$ \\
\hline & & $49-88$ & $49-88$ & $50-88$ \\
\hline & Verbal IQ $^{\mathrm{a}}$ & $97.08(11.52)$ & $97.30(11.67)$ & $96.14(11.68)$ \\
\hline & & $80-122$ & $80-122$ & $86-119$ \\
\hline & Nonverbal $\mathrm{IQ}^{\mathrm{b}}$ & $113.27(13.93)$ & $115.17(14.23)$ & $105.14(9.53)$ \\
\hline & & $83-141$ & $83-141$ & $97-123$ \\
\hline & SCQ total score ${ }^{c}$ & $23.16(7.50)$ & $23.40(7.96)$ & $22.14(5.55)$ \\
\hline & & $11-36$ & $11-36$ & $11-28$ \\
\hline & ADI-R total score ${ }^{\mathrm{d}}$ & $26.57(8.59)$ & $27.03(9.31)$ & $24.57(4.28)$ \\
\hline & & $13-46$ & $13-46$ & $20-30$ \\
\hline \multirow{14}{*}{ Time 2} & Age (months) & $100.84(11.15)$ & $99.07(10.64)$ & $104.71(14.08)$ \\
\hline & & $80-119$ & $80-118$ & $81-119$ \\
\hline & Verbal IQ $^{\mathrm{a}}$ & $93.89(17.88)$ & $93.57(19.07)$ & $94.86(9.03)$ \\
\hline & & $62-138$ & $62-138$ & $83-106$ \\
\hline & Nonverbal IQ ${ }^{\mathrm{b}}$ & $104.35(12.72)$ & $105.10(13.47)$ & $101.14(8.93)$ \\
\hline & & $80-135$ & $80-135$ & $91-115$ \\
\hline & SCQ total score ${ }^{c}$ & $14.97(6.14)^{\mathrm{c}}$ & $16.76(5.32)$ & $7.97(2.94)$ \\
\hline & & $2-25$ & $5-25$ & $2-11$ \\
\hline & ADOS-G total score & $12.62(4.98)$ & $14.23(4.06)$ & $5.71(.95)$ \\
\hline & & $4-23$ & $7-23$ & $4-6$ \\
\hline & Age first received & $39.17(13.43)$ & $41.90(13.12)$ & $27.86(7.92)$ \\
\hline & intervention (months) & $16-66$ & 21-66 & $16-36$ \\
\hline & Intervention received & $2.84(3.59)$ & $2.95(3.73)$ & $2.38(3.17)$ \\
\hline & $\begin{array}{l}\text { during } 3 \text {-year period } \\
\text { (mean hours per week) }\end{array}$ & $0-15$ & $0-15$ & $0-9.33$ \\
\hline
\end{tabular}

Note. ASD = autism spectrum disorder; $\mathrm{SCQ}=$ Social Communication Questionnaire; ADI-R = Autistic Diagnostic Interview-Revised; ADOS-G = Autism Diagnostic

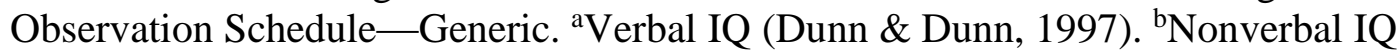
(Roid \& Miller, 1997). ${ }^{\mathrm{c} S C Q}(n=36)$. ${ }^{\mathrm{d}}$ Elevated scores reflect increased symptomatology. 
Table 2

Pearson Correlations and Partial Correlations for Longitudinal Relationships Within and Between Domains on the Social Communication Questionnaire (SCQ; Rutter et al., 2003)

\section{Time 2}

Social Communication $\begin{aligned} & \text { Repetitive } \\ & \text { behaviors }\end{aligned}$

$\underline{\text { Time } 1}$

Full $(d f=36)$

Social

$.73 * * *$

$.58 * * *$

$.38 * *$

Communication $\quad .29$

$.38 * *$

.29

Repetitive behaviors

.25

.20

$.54 * * *$

Verbal

and nonverbal,

IQ partialed

Social

$.70 * * *$

$.53 * * *$

$.38^{*}$

$(d f=32)$

Communication

$.42 * *$

$.38^{*}$

.30

Note. ${ }^{*}$ (Marginally) significant at the .05 level. **Significant at the .01 level.

.16

$.56 * * *$

***Significant at the .001 level. 